\title{
Antimicrobial Colloidal Silver-Lignin Particles via Ion and Solvent Exchange
}

\author{
Kalle Lintinen, ${ }^{\dagger}$ Sanna Luiro, ${ }^{\dagger}$ Patrícia Figueiredo, ${ }^{\ddagger}$ Ekaterina Sakarinen, ${ }^{\dagger}$ Zekra Mousavi, $^{\perp}$
} Jani Seitsonen, ${ }^{\#}$ Guillaume N. S. Rivière, ${ }^{\nabla}{ }^{\mathbb{C}}$ Ulriika Mattinen, ${ }^{\perp}$ Matti Niemelä, ${ }^{\bigcirc}$ Päivi Tammela, ${ }^{\circledR}$ (i) Monika Österberg, ${ }^{\nabla}{ }^{\odot}$ Leena-Sisko Johansson, ${ }^{\nabla}$ Johan Bobacka, ${ }^{\perp}$ Hélder A. Santos, ${ }^{\ddagger}, \|_{\odot}$ and Mauri A. Kostiainen ${ }^{*} \dagger+0$

${ }^{\dagger}$ Biohybrid Materials, Department of Bioproducts and Biosystems, Aalto University, Kemistintie 1, 02150 Espoo, Finland

${ }^{*}$ Drug Research Program, Division of Pharmaceutical Chemistry and Technology, Faculty of Pharmacy, ${ }^{\S}$ Centre for Drug Research, Division of Pharmaceutical Biosciences, Faculty of Pharmacy, and "Helsinki Institute of Life Science, HiLIFE, University of Helsinki, FI-00014 Helsinki, Finland

${ }^{\perp}$ Laboratory of Analytical Chemistry, Johan Gadolin Process Chemistry Centre, Åbo Akademi University, Biskopsgatan 8, FI-20500 Turku, Finland

${ }^{\#}$ Nanomicroscopy Center, Department of Applied Physics, School of Science, Aalto University, P.O. Box 15100, FI-00076 Espoo, Finland

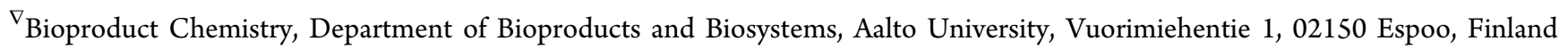

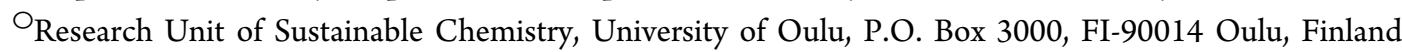

Supporting Information

ABSTRACT: Acid-precipitated lignin nanoparticles with a cationic polymer coating exhibit antibacterial activity when infused with silver. While the use of such particles would be beneficial due to their high antibacterial activity with a low silver content, their production holds steps that are difficult to scale up to inexpensive industrial manufacture. For example, the production of acid-precipitated lignin nanoparticles requires the use of ethylene glycol, which is not easily recycled. Furthermore, the binding of silver to these particles is weak, and thus the particles need to be used rapidly after preparation. Here, we show that with a deprotonation reaction of an organic solution of anhydrous lignin and subsequent ion

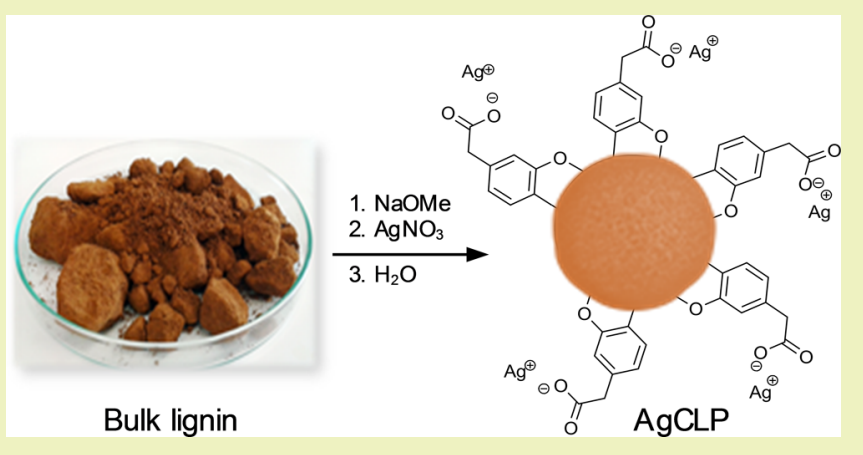
exchange with silver nitrate and colloid formation by solvent exchange, highly spherical silver carboxylate colloidal lignin particles (AgCLPs) can be prepared. Silver is not released from the particles in deionized water but can be released in physiological conditions, shown by their high antibacterial efficacy with low silver loading. In comparison to lignin nanoparticles with weakly bound silver, AgCLPs have high antibacterial activity even without cationic polyelectrolyte coating, and they retain their antibacterial activity for days. While the rapid depletion of silver from silver-infused lignin nanoparticles can be considered beneficial for some applications, the sustained antibacterial activity of the AgCLPs with ionically bound silver will enable their use in applications where silver nanoparticles have been previously used. Our results demonstrate that CLPs, which can be produced with a closed cycle process on a large scale, can be rapidly and quantitatively functionalized into active materials.

KEYWORDS: lignin, colloid, silver, antibacterial, particle

\section{INTRODUCTION}

Lignin is the most common renewable source of aromatic groups on Earth and an attractive biopolymer with potential for the development of new materials. ${ }^{1}$ Besides its industrial applications, ${ }^{2,3}$ lignin has also been used to produce different types of lignin nanoparticles for antimicrobial and drug delivery applications. ${ }^{4-6}$ Their common preparation methods include anti-solvent precipitation, interfacial cross-linking, polymerization, solvent exchange, and sonication. ${ }^{1,7,8}$
While silver nanoparticles (AgNPs) are efficient antimicrobial, antifungal, and antiviral agents, their widespread use is limited by their persistence in nature and negative effects relating to their bioaccumulation in water systems. ${ }^{9}$ Richter et al. reported a method for infusing lignin nanoparticles with silver ions and showed that these environmentally benign

Received: May 6, 2019

Revised: August 15, 2019

Published: August 16, 2019 
lignin-core nanoparticles (EbNPs) could be used similarly to AgNPs, with a smaller loading of silver and with no persistence of silver in the nanoparticles after their use. ${ }^{4}$ While their method could be applied into large-scale antibacterial use, several issues hinder its industrial application, such as the recovery of the solvent ethylene glycol, weak binding of $\mathrm{AgNO}_{3}$ into the particles, and the fast release of silver from the particles.

We have recently shown that highly spherical colloidal lignin particles (CLPs) can be prepared with a three-solvent polarity exchange method, which recycles all of the solvents, with a cost of production of $1.0 € / \mathrm{kg}$ of dry lignin particles produced. ${ }^{10-12}$ This method is adaptable to lignin functionalization, especially when the reactions are quantitative. While our method of producing CLPs would be suitable for silver infusion with the Richter method, we consider the non-quantitative binding of silver ions into CLPs problematic both for the large-scale production of silver-infused lignin particles and for the stability of such particles under storage. A solution to this is to ionically bind silver into CLPs. We have previously shown that the reaction of iron isopropoxide with lignin in anhydrous tetrahydrofuran (THF) yields a variety of metal-lignin structures, depending on the metal alkoxide to acidic functional group (phenolic $\mathrm{OH}$ or carboxylic acid) ratio. ${ }^{13}$ Recently, Nypelö et al. used lignin as an emulsifier to reduce silver nitrate with $\mathrm{NaBH}_{4}$ to produce silver-loaded lignin particles. ${ }^{14}$ Here, we show that in a similar approach, the acidic (carboxylic and phenolic) groups of lignin can be deprotonated with sodium methoxide ( $\mathrm{NaOMe}$ ) to yield corresponding sodium carboxylates and phenolates. Silver can then be ionically bound to these sites via ion exchange with $\mathrm{AgNO}_{3}$. However, only silver carboxylate is both stable in water and enables the formation of uniform spherical CLPs from the anhydrous solution. Spherical silver carboxylate CLPs (AgCLPs) are self-assembled by inserting an anhydrous solution of silver carboxylate of lignin in THF/methanol $(\mathrm{MeOH})$ into water, producing AgCLPs with sizes ranging from $\sim 60$ to $200 \mathrm{~nm}$, depending on the lignin concentration and the degree of lignin deprotonation. The silver carboxylate AgCLPs do not release silver when no countercations are present, making them stable even as aqueous dispersions. However, the AgCLPs show clear and strong antibacterial activity in physiological conditions, indicating the release of silver ions.

The particles are antimicrobial toward Gram-negative bacteria, such as Escherichia coli, with a minimal inhibitory concentration, even without a coating with positive polyelectrolyte. Due to the stronger binding of silver as carboxylates in AgCLPs, they have a significantly longer time of activity, making them suitable for many applications, where the silver release from silver-infused lignin nanoparticles is too rapid.

\section{EXPERIMENTAL SECTION}

Chemicals. The LignoBoost softwood kraft lignin was BioPivo (UPM), with a solid content of 68.1 wt $\%$ as measured by thermogravimetric analysis (TGA) and ash content of $\sim 1$ wt \%. Silver nitrate $\left(\mathrm{AgNO}_{3}\right)$ and $\mathrm{NaOMe}$ were purchased from Aldrich, and molecular sieves $(0.3 \mathrm{~nm})$ were from Merck. THF and methanol were obtained from VWR. All solvents were further dried using molecular sieves. All work with $\mathrm{AgNO}_{3}$ was conducted in a darkened room.

Preparation of Anhydrous Reagent Solutions. Lignin was dried in a vacuum oven at $80{ }^{\circ} \mathrm{C}$ overnight to remove most of the water. Dry lignin was dissolved in anhydrous THF after which the insoluble ash was separated from the lignin solution by $20 \mathrm{~min}$ centrifugation at $4000 \mathrm{~g}$. The THF solution was diluted with a 1:1 mass ratio of $\mathrm{MeOH}$ to THF after which molecular sieves were added to the solution. $\mathrm{NaOMe}$ (95\% purity) was dissolved in anhydrous $\mathrm{MeOH}$ by shaking and sonication. The $\mathrm{MeOH}$ solution was diluted with a 1:1 mass ratio of THF to $\mathrm{MeOH}$. No molecular sieves were added as any water in the solution will react first with $\mathrm{NaOMe}$ to produce $\mathrm{NaOH}$ and $\mathrm{MeOH}$. Care was taken to prevent the introduction of water into the solution. $\mathrm{AgNO}_{3}$ was dissolved in anhydrous $\mathrm{MeOH}$ by shaking and sonication. The undissolved $\mathrm{Ag}_{2} \mathrm{O}$ / $\mathrm{Ag}$ precipitate present in the solution was separated from the solution by $20 \mathrm{~min}$ centrifugation at $4000 \mathrm{~g}$. The mass of the precipitate was weighed to be less than $1 \mathrm{wt} \%$ of the $\mathrm{AgNO}_{3}$ mass. The $\mathrm{MeOH}$ solution was diluted with a 1:1 mass ratio of THF to $\mathrm{MeOH}$ after which molecular sieves were added to the solution.

Lignin Deprotonation and Ion Exchange. Sodium carboxylate/phenolate lignin ( NaLignin) was prepared by adding $\mathrm{NaOMe}$ solution into anhydrous lignin solution in a vial under constant stirring. The deprotonation, that is, the conversion of carboxylic acid and phenolic groups into sodium carboxylate and phenolate groups, was considered to occur instantaneously. The deprotonated lignin was immediately used either for the preparation of AgLignin or NaCLPs. AgLignin was prepared by adding anhydrous $\mathrm{AgNO}_{3}$ solution into NaLignin solution under constant stirring. The ion exchange, that is, the conversion of sodium carboxylate/phenolate into silver carboxylate/phenolate, was considered to occur instantaneously.

Formation of Colloidal Lignin Particles. Organic lignin solutions of either NaLignin or AgLignin were rapidly inserted into water (minimum final water content of $75 \mathrm{wt} \%$ ) in a $100 \mathrm{~mL}$ roundbottom flask, under constant stirring, causing lignin to rapidly selfassemble into spherical colloidal particles. The organic solvents were removed by rotary evaporation at $40{ }^{\circ} \mathrm{C}$ immediately after preparation. Depending on the experiments, the colloidal lignin particles were used as they were or further processed (coated with poly(diallyldimethylammonium chloride) (PDAC) and/or dialyzed against water). Aqueous $\mathrm{AgCLPs}$ were inserted into a dialysis tube (12-14 kDa MWCO) and dialyzed against (periodically changed) deionized water. Besides water-soluble compounds, such as the side product $\mathrm{NaNO}_{3}$, dialysis also removed all free $\mathrm{Ag}$ or $\mathrm{Ag}_{2} \mathrm{O}$ NPs smaller than the pore size of the dialysis membrane.

PDAC Coating of AgCLPs. Aqueous PDAC (Mw 100-350 kDa, Polysciences, USA) was added into aqueous AgCLPs under constant stirring with a $5 \mathrm{wt} \%$ PDAC to AgCLP ratio. PDAC coating was considered to occur instantaneously. The surface charge modification of the lignin nanoparticles was monitored by zeta potential measurements.

Particle Dispersion Characterization. The mean particle size and electrophoretic mobility of the lignin samples were measured using a Malvern Zetasizer Nano-ZS90 Instrument (UK). Experiments were performed with duplicate samples when necessary, with $\sim 50 \mathrm{~g}$ $\mathrm{L}^{-1}$ aqueous dispersions. Intensity-based mean average particle sizes were used in analysis and reporting of the data.

${ }^{31}$ P-NMR. The chemical composition of lignin was determined using ${ }^{31} \mathrm{P}$-NMR with a procedure based on the method by Granata and Argyropoulos. ${ }^{15}$ A lignin sample was accurately weighed $(25 \mathrm{mg}$ ) and dissolved in $150 \mu \mathrm{L}$ of $N, N$-dimethylformamide in a $2 \mathrm{~mL}$ vial. After complete dissolution, $100 \mu \mathrm{L}$ of pyridine, $200 \mu \mathrm{L}(0.05 \mathrm{M})$ of internal standard solution (ISTD) of endo- $N$-hydroxy-5-norbornene2,3-dicarboximide (e-HNDI, $0.01 \mathrm{mmol}$ ) in pyridine $/ \mathrm{CDCl}_{3}(1.6 / 1$, $\mathrm{v} / \mathrm{v})$, and $50 \mu \mathrm{L}$ of the $\operatorname{Cr}(\mathrm{acac})_{3}$ solution $\left(11.5 \mathrm{mg} \mathrm{mL}^{-1}\right)$ in pyridine $/ \mathrm{CDCl}_{3}(1.6 / 1, \mathrm{v} / \mathrm{v})$ were added. Then, $200 \mu \mathrm{L}$ of the phosphitylation reagent 2-chloro-4,4,5,5-tetramethyl-1,3,2-dioxaphospholane [P.R.(II)] was added dropwise. Finally, $300 \mu \mathrm{L}$ of $\mathrm{CDCl}_{3}$ was added to the solution, and a clear brown to black solution was achieved. Dissolution was aided by slow stirring with a magnetic stirrer and gentle heating at $45{ }^{\circ} \mathrm{C}$. Freshly prepared samples were measured with ${ }^{31} \mathrm{P}-\mathrm{NMR}$ immediately at room temperature. A Bruker $500 \mathrm{MHz}$ NMR spectrometer was used for the measurement. Chemical shifts are reported relative to the sharp signal (132.2 ppm) 


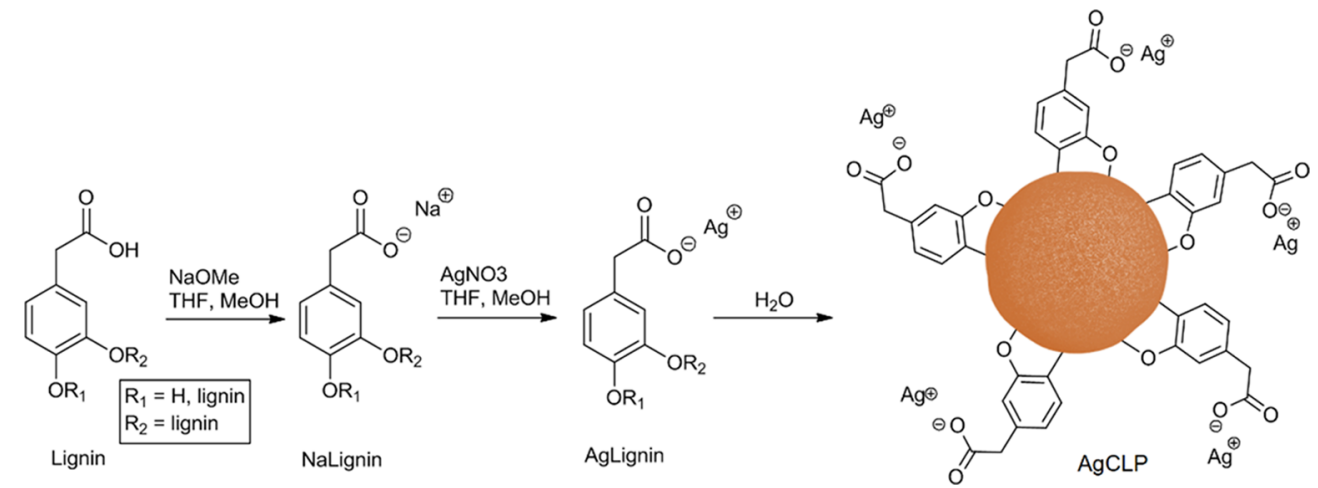

${ }^{a}$ Only a single carboxylic acid-bearing aromatic group of the complex polymeric lignin molecule is shown to illustrate the effect of the deprotonation and ion exchange reactions.

originating from the reaction between water and P.R.(II). The following NMR parameters were used: 512 scans, 2 s pulse delay, and $90^{\circ}$ pulse.

Transmission Electron Microscopy (TEM). TEM was performed on an FEI Tecnai 12 (USA) operating at $120 \mathrm{kV}$. Water dispersions were applied on a carbon film support grid and incubated for $2 \mathrm{~min}$, and excess water was removed by blotting the side of the grid onto filter paper. Imaging was done in the bright-field mode with slight underfocus.

Energy-Dispersive X-ray Spectroscopy (EDS). TEM-EDS measurements were performed on an analytical HR-TEM JEOL JEM-2800 operated at $200 \mathrm{kV}$ with high-speed EDS.

Inductively-Coupled Plasma Optical Emission Spectroscopy (ICP-OES). Before the measurement, samples were homogenized using an ultrasonic bath. After ultrasonication, $100 \mathrm{mg}$ of sample was weighed into a polyethylene tube, $0.5 \mathrm{~mL} 65 \%(\mathrm{v} / \mathrm{v}) \mathrm{HNO}_{3}$ was added, and samples were diluted to $5 \mathrm{~mL}$ with ultrapure water. After that, a Perkin Elmer Optima 5300 DV ICP-OES, which allows either an axial or radial mode of viewing of the plasma, was used to determine $\mathrm{Ag}(328.068,338.289 \mathrm{~nm})$. The ICP-OES instrument was equipped with an AS-93plus autosampler, a Ryton double-pass Scotttype spray chamber, and a Gem Tip cross-flow pneumatic nebulizer. The instrumental parameters for ICP-OES were as follows: RF power of $1.3 \mathrm{~kW}$, nebulizer gas flow of $0.8 \mathrm{~L} \mathrm{~min}^{-1}$, auxiliary gas flow of $0.2 \mathrm{~L}$ $\mathrm{min}^{-1}$, plasma gas flow of $15 \mathrm{~L} \mathrm{~min}^{-1}$, and sample uptake of $1.5 \mathrm{~mL}$ $\mathrm{min}^{-1}$. Normal resolution and axial mode of viewing were used in the measurements. All ICP-OES measurements were performed following the guidelines described in the SFS-EN ISO 11885 standard. ${ }^{17}$

Silver Ion $\left(\mathrm{Ag}^{+}\right)$Measurement Using Silver lon-Selective Electrode (Ag-ISE). Description of the method can be found in the Supporting Information.

X-ray Photoelectron Spectroscopy (XPS). Description of the method can be found in the Supporting Information.

Antibacterial Experiments. Antimicrobial activity was evaluated with CLSI (formerly NCCLS) broth microdilution protocols for antimicrobial testing, and the assays were carried out in triplicate. ${ }^{16}$ The AgCLPs used in the antibacterial experiments were prepared via AgLignin as shown in Scheme 1, with $2.6 \%$ of the $\mathrm{PhOH} / \mathrm{COOH}$ groups deprotonated and ion-exchanged. The Supporting Information also shows data on samples with $15 \%$ of the $\mathrm{PhOH} / \mathrm{COOH}$ groups deprotonated and ion-exchanged with the same method, samples of $\mathrm{NaCLPs}$ infused with $\mathrm{AgNO}_{3}$, both with $2.6 \%$ and $15 \%$ deprotonation and ion exchange, and all of the above samples coated with PDAC. E. coli ATCC 25922, Pseudomonas aeruginosa ATCC 27853, and Staphylococcus aureus ATCC 29213 were obtained from Microbiologics Inc. (St. Cloud, MN). For the assays, bacteria were grown in Mueller-Hinton broth (MHB, Becton Dickinson, Sparks, MD) overnight at $37{ }^{\circ} \mathrm{C}$. Dialyzed CLPs, CLPs-PDAC, AgCLPs via AgLignin and AgCLPs-PDAC (15 and 2.6\%), Ag-infused NaCLPs and NaCLPs-PDAC (15 and 2.6\%) suspensions, $\mathrm{AgNO}_{3}$ dilutions in
MHB, and the controls were added to 96-well plates (Thermo Fisher Scientific, Roskilde, DK), and the bacterial suspensions were then seeded into the plates at a final concentration of $5 \times 10^{5}$ colony forming units $(\mathrm{CFU}) \mathrm{mL}^{-1}$ in MHB. In addition, each plate carried a negative control (MHB) and ciprofloxacin as positive control at $\mathrm{MIC}_{90}$ (E. coli, $0.02 \mu \mathrm{g} \mathrm{mL}^{-1}$; P. aeruginosa, $1 \mu \mathrm{g} \mathrm{mL}^{-1}$; and S. aureus, $\left.0.5 \mu \mathrm{g} \mathrm{mL}^{-1}\right)$. Then, the plates were incubated for $24 \mathrm{~h}$, and the absorbance was measured at $620 \mathrm{~nm}$ on a Multiskan GO UV-vis spectrophotometer (Thermo Fisher Scientific, Finland). The percentage of inhibition was calculated by comparing the average optical density at $620 \mathrm{~nm}\left(\mathrm{OD}_{620}\right)$ of the bacterial suspension with and without the compound (negative control).

\section{RESULTS AND DISCUSSION}

Formation of Colloidal Silver-Lignin Particles. We chose LignoBoost softwood kraft lignin as the source of lignin as it is currently produced in a scale of tens of thousands of tons per year, both in Europe and in the USA. ${ }^{18}$ We have shown previously that highly spherical colloidal lignin particles (CLPs) can be assembled from lignin via a solvent exchange method. ${ }^{19}$ LignoBoost lignin is highly soluble in THF and can produce CLPs upon addition into water at a concentration of at least $2.8 \mathrm{wt} \%$ with the use of a co-solvent, such as ethanol. ${ }^{11}$ The used solvents can be separated and recovered from the particles by ultrafiltration and by distillation. The recovered solvents can be used to further dissolve lignin, enabling a closed cycle process. It was shown that the energy consumption of the process is minimal. ${ }^{12}$

We found that the carboxylic acid moieties of kraft lignin (UPM BioPiva, purified with the LignoBoost process) can be deprotonated with $\mathrm{NaOMe}$ and ion-exchanged with silver, without disrupting the CLP self-assembly. Scheme 1 shows the pathway for the formation of silver carboxylate CLPs (AgCLPs). The formation of AgCLPs starts with the formation of NaLignin by deprotonating an anhydrous solution of lignin with sodium methoxide $(\mathrm{NaOMe})$ dissolved in a mixture of $\mathrm{THF}$ and methanol $(\mathrm{MeOH})$.

The solvent mixture was chosen as both lignin and $\mathrm{NaOMe}$ are soluble in it, while only lignin is sufficiently soluble in neat $\mathrm{THF}$ and $\mathrm{NaOMe}$ in neat $\mathrm{MeOH}$. Silver carboxylate lignin (AgLignin) is formed by ion exchange of NaLignin with $\mathrm{AgNO}_{3}$, both again in THF/MeOH. As the $\mathrm{p} K_{\mathrm{a}}$ values of the carboxylic acid groups of lignin are below 4.6 and most of the $\mathrm{p} K_{\mathrm{a}}$ values of the phenolic groups lie above 9 (with some species with electron-withdrawing groups having a $\mathrm{p} K_{\mathrm{a}}$ value between 7.3 and 9), it can be observed that there is almost no 

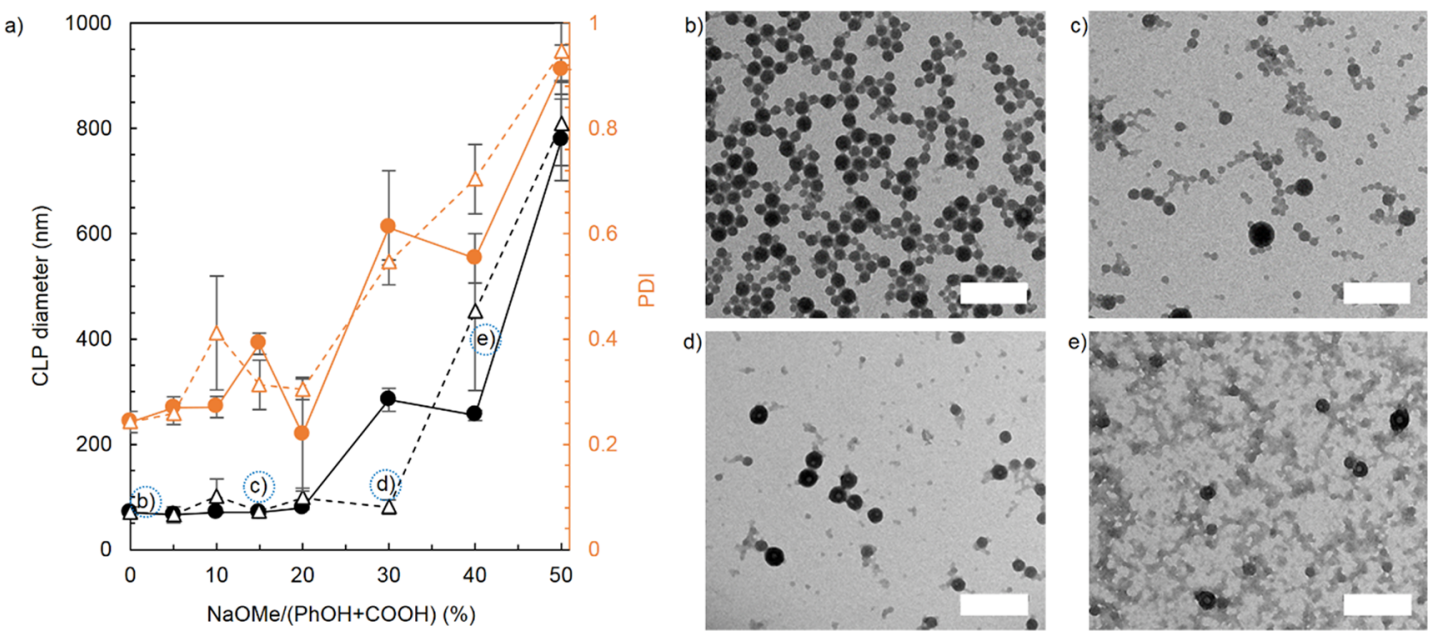

Figure 1. (a) Diameter (black) and polydispersity (PDI, orange) of NaCLPs (circles) and AgCLPs (triangles) as a function of the degree of lignin deprotonation, determined by the ratio of $\mathrm{NaOMe}$ to the acidic $\mathrm{OH}$ groups $(\mathrm{COOH}$ and $\mathrm{PhOH}$ ) in the used lignin. (All data points are an average of two independent samples with an error bar presenting the data range.) TEM images of AgCLPs with varying degrees of deprotonation/ion exchange: (b) 2.6, (c) 15, (d) 30, and (e) 40\%. The letters in (a) correspond to TEM images (b)-(e). Scale bar of $200 \mathrm{~nm}$.

overlap between the deprotonation of carboxylic acid and phenolic groups of lignin. ${ }^{20}$ The degree of deprotonation is determined as the ratio of the reacted $\mathrm{NaOMe}$ versus the carboxylic acid and phenolic groups of lignin, as determined by ${ }^{31} \mathrm{P}$ NMR. In the anhydrous organic medium, the reaction can be assumed quantitative, whereas in aqueous media, the presence of an equimolar concentration of $\mathrm{Na}^{+}$ions to $\mathrm{OH}^{-}$ ions (determined by the $\mathrm{pH}$ of the dispersion) slightly reduces the $\mathrm{Na}^{+}$ion concentration of the CLPs in alkaline $\mathrm{pH}$. In the case of the $\mathrm{pH}$ range of sodium carboxylate (from $\mathrm{pH} 2.5$ to 6.5 with 1 to $99 \%$ deprotonation), the $\mathrm{OH}^{-}$and subsequently $\mathrm{Na}^{+}$concentrations are small enough to be neglected. IR spectroscopy and X-ray photoelectron spectroscopy (XPS) show that AgLignin is present fully in the silver carboxylate form when the degree of deprotonation and ion exchange is well below the $\mathrm{COOH}$ content of the used lignin (see the Supporting Information).

AgCLPs self-assemble when the organic AgLignin solution is introduced into stirred water. It is also possible to self-assemble NaLignin straight into sodium carboxylate CLPs (NaCLPs) and conduct the ion exchange with aqueous $\mathrm{AgNO}_{3}$.

The effects of the degree of deprotonation of acidic (carboxylic and phenolic) groups of NaLignin and the degree of deprotonation/ion exchange of AgLignin on the CLP diameter and polydispersity (PDI) are shown in Figure 1a. To prevent the precipitation of the less soluble AgLignin from the $\mathrm{MeOH} / \mathrm{THF}$ mixture, the self-assembly was conducted with a low concentration of lignin solution $(2.1 \mathrm{mg} / \mathrm{g})$, resulting in CLPs with a lignin concentration of $0.51 \mathrm{mg} / \mathrm{g}$. NaCLPs have a diameter of $\sim 60 \mathrm{~nm}$ and polydispersity index (PDI) of $\sim 0.2$ up to $\sim 20 \%$ deprotonation, as determined by dynamic light scattering (DLS). These values are comparable to those measured from sodium-free CLPs. Beyond this point, both the $\mathrm{NaCLP}$ diameter and PDI start to increase. Our assumption is that the higher solubility of lignin due to the dissociation of the phenolic $\mathrm{OH}$ groups disrupts the self-assembly of CLPs. The self-assembly of AgCLPs both in terms of particle diameter and PDI is very similar to that of NaCLPs. This indicates that the effect of the countercation in the carboxylate and phenolate lignin does not influence the amphiphilic nature of lignin. However, at higher lignin concentrations, AgLignin begins to precipitate out of the $\mathrm{MeOH} / \mathrm{THF}$ solution as soon as the $\mathrm{NaOMe}$ and $\mathrm{AgNO}_{3}$ loading is high enough to produce silver phenolate.

The DLS data on AgCLP formation already indicates that good-quality AgCLPs cannot be produced with a significant quantity of the silver phenolate form of lignin. An even clearer indication of the challenges with silver phenolate lignin can be seen with TEM and EDS (see Figure S2). The AgCLPs formed from AgLignin with 2.6 and 15\% deprotonation/ion exchange (Figures $1 \mathrm{~b}, \mathrm{c}, 1.0$ and $5.8 \mathrm{wt} \% \mathrm{Ag}$ of CLP, respectively) are moderately dispersed and extremely spherical. With $30 \%$ ion exchange and deprotonation (Figure 1d), AgCLP polydispersity starts to increase, but rather small particles are formed when the AgLignin concentration is kept low. However, at $40 \%$ ion exchange and deprotonation (Figure 1e), even with rather dilute conditions, the AgCLPs are largely aggregated, indicating that the CLP formation is disrupted. With EDS, it is possible to estimate the silver content of the AgCLPs by comparing the Ag peak of individual particles to their $\mathrm{S}$ peak, known to be $3.10 \mathrm{wt} \%$ of the used lignin mass. ${ }^{19}$ The $\mathrm{Ag}$ content of AgCLPs prepared via AgLignin with $1.0 \mathrm{wt} \% \mathrm{Ag}$ to lignin showed an Ag content of 1.0 wt \% also with EDS. However, when the degree of deprotonation and ion exchange was raised above the degree of $\mathrm{COOH}$ content of lignin, EDS showed that the detected silver content no longer matched the calculated silver content despite the good particle quality by DLS. We assume this to be due to the unstable nature of the silver phenolate groups, which are possibly hydrolyzed releasing silver hydroxide, which can further react to silver oxide. ${ }^{21}$ In EDS, these AgCLPs with higher silver loadings show a non-uniform distribution of silver and also the presence of silver(oxide) particles (the presence of oxygen in the particles cannot be determined from the EDS spectra).The total silver content and the free silver ion content of the AgCLPs were measured by ICP-OES and the Ag-ISE, respectively (Table 1). The amount of silver detected by ICP-OES before dialysis matched closely with the calculated silver content and was considered as the reference value. When the silver content is kept below the $\mathrm{COOH}$ content, ICP-OES showed that there was almost no loss in the silver content of the AgCLPs, which was also confirmed by EDS. However, 
Table 1. Effect of the Degree of Acidic Hydroxyl (Phenol/ Carboxylic Acid) Deprotonation/Ion Exchange on the Total Ag Content of the Dialyzed AgCLPs and the Free Ag Content of the AgCLPs before Dialysis As Measured by ICP-OES and Ag-ISE, Respectively

$\begin{array}{cccc}\begin{array}{c}\text { degree of } \mathrm{PhOH} / \mathrm{COOH} \\ \text { deprotonation and ion } \\ \text { exchange (\%) }\end{array} & \begin{array}{c}\text { Ag content } \\ \text { of lignin } \\ \text { (wt \%) }\end{array} & \begin{array}{c}\text { total silver } \\ \text { after dialysis } \\ (\%)\end{array} & \begin{array}{c}\text { free silver } \\ \text { before } \\ \text { dialysis (\%) }\end{array} \\ 2.6 & 1.0 & 94 & 0 \\ 15 & 5.8 & 44 & 0\end{array}$

when the degree of deprotonation/ion exchange was raised above the $\mathrm{COOH}$ content, the total silver content of the dialyzed AgCLPs dropped. This matches with the observations with EDS. As expected, no free silver was detected using the Ag-ISE as the silver in the AgCLPs is ionically bound to the CLPs. The Ag-ISE was also used to potentiometrically study the silver consumption of NaCLPs (Figure 2). An equal

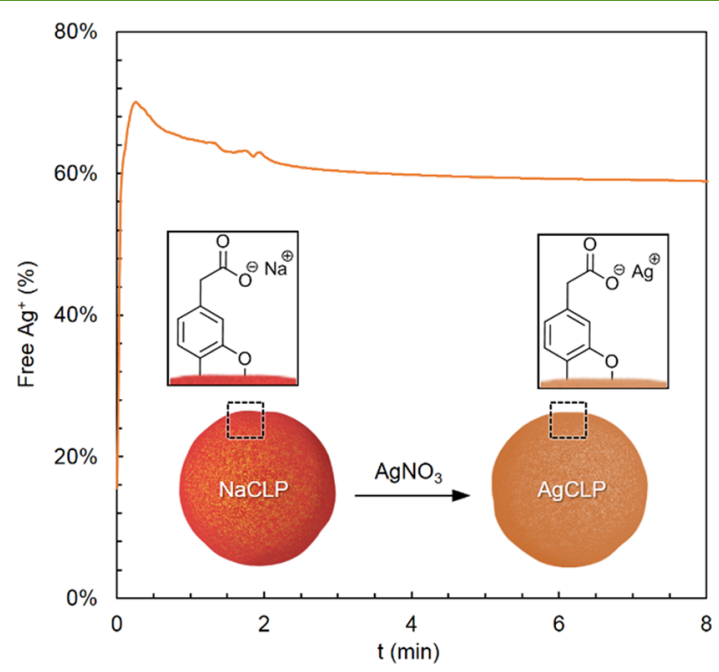

Figure 2. $\mathrm{AgNO}_{3}$ consumption of NaCLPs, measured in a potentiometric cell using a silver selective electrode (Ag-ISE), determined by the loss of $\mathrm{Ag}^{+}$signals. The value at time $0 \mathrm{~min}$ corresponds to the silver concentration at the point of addition of $\mathrm{AgNO}_{3}$ solution into the NaCLP dispersion.

volume of aqueous $\mathrm{AgNO}_{3}$ was added to $\mathrm{NaCLPs}$, with the $\mathrm{Na}$ concentration of NaCLPs matching the $\mathrm{AgNO}_{3}$ concentration
(0.71 mmol/L after mixing). In $15 \mathrm{~s}$, the free silver concentration rose to $70 \%$ and settled to the final concentration of $59 \%$ in less than $5 \mathrm{~min}$. This can be attributed to a fast ion exchange at or near the surface of the $\mathrm{NaCLPs}$. Apparently, the diffusion of Ag into the core of the AgCLPs is a much slower process than could be observed with this method. It was observed that non-deprotonated CLPs also bind $\mathrm{AgNO}_{3}$, although to a slightly lesser extent ( $84 \%$ of the binding) than the NaCLPs. This is explained by the presence of residual sodium in the LignoBoost lignin after purification. ${ }^{22}$ It was also observed that when the measuring cell (with AgISE) was not carefully protected from light, additional $\mathrm{AgNO}_{3}$ reduction was observed (Figure S4), possibly caused by the sodium carboxylate NaCLPs catalyzing the photoreduction of silver ions into silver nanoparticles. ${ }^{23}$

Antibacterial Activity of AgCLPs. The antibacterial activity of AgCLPs was initially tested immediately after preparation. However, it was confirmed that the particles are stable and can be used at least for several days after preparation, which is expected of ionic binding of $\mathrm{Ag}^{+}$to CLPs. Quantitative antibacterial tests were conducted on two Gram-negative bacteria, E. coli (ATCC 25922) and $P$. aeruginosa (ATCC 27853), as well as on Gram-positive S. aureus (ATCC 29213).

Testing was carried out according to a standardized exposure protocol based on the broth microdilution method according to the CLSI guidelines. ${ }^{16}$ The absorbance at $620 \mathrm{~nm}$ was measured after $24 \mathrm{~h}$ of incubation of the bacteria with the particle suspension and compared with control samples. The AgCLP's efficiency was compared against $\mathrm{AgNO}_{3}$ solutions (Figure 3).

The $\mathrm{Ag}^{+}$content of the samples incubated with E. coli ranged between 2.5 and $10 \mathrm{mg} \mathrm{L}^{-1}$, whereas for $P$. aeruginosa and $S$. aureus, the $\mathrm{Ag}^{+}$content ranged from 2.5 to $30 \mathrm{mg} \mathrm{L}^{-1}$. The concentration of AgCLPs and respective inhibition of growth against the three bacteria strains used for the experiments are detailed in Table S1.

After the AgCLP self-assembly, the particles were dialyzed against distilled water to remove any weakly bound silver. The AgCLPs exhibited a strong antimicrobial activity against Gramnegative $E$. coli at $5 \mathrm{mg} \mathrm{L}^{-1} \mathrm{Ag}^{+}$content, with $90 \%$ inhibition of growth after $24 \mathrm{~h}$ of exposure, which was comparable to the free $\mathrm{AgNO}_{3}$ solution at $5 \mathrm{mg} \mathrm{L} \mathrm{mg}^{+}$content (Figure 3a). Tests with a higher degree of ion exchange than the a)

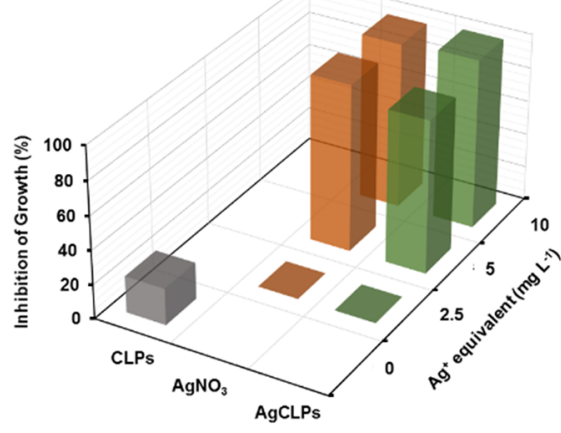

b)

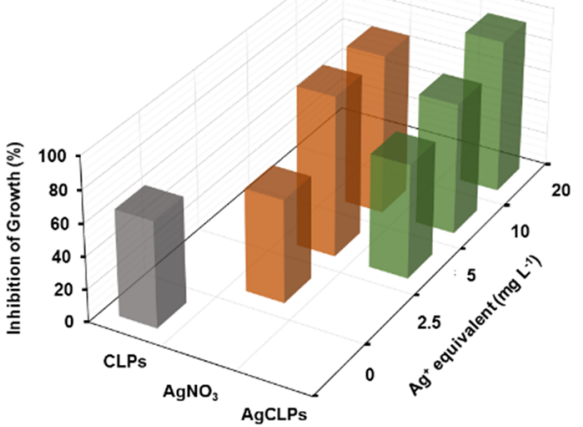

c)

Staphylococcus aureus (ATCC 29213)

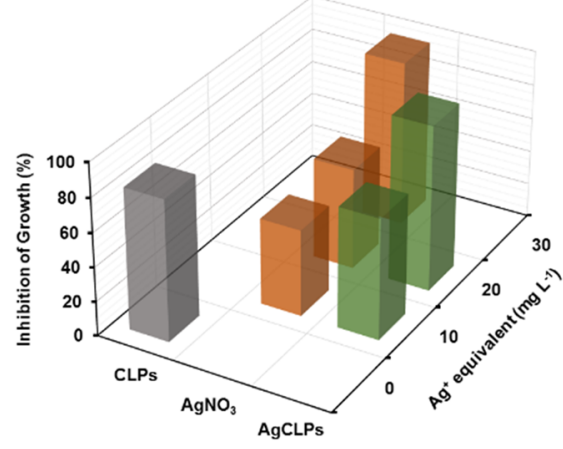

Figure 3. Quantification of inhibition of growth as a function of $\mathrm{Ag}^{+}$equivalent $\left(\mathrm{mg} \mathrm{L}^{-1}\right)$ of $\mathrm{AgCLPs}$ and control samples on CLPs (equivalent lignin mass of AgCLPs to $10 \mathrm{mg} \mathrm{L}^{-1}$ of $\mathrm{Ag}^{+}$for E. coli and $20 \mathrm{mg} \mathrm{L}^{-1}$ of $\mathrm{Ag}^{+}$for P. aeruginosa and S. aureus) and $\mathrm{AgNO}_{3}$ after $24 \mathrm{~h}$ exposure: (a) E. coli (ATCC 25922); (b) P. aeruginosa (ATCC 27853), and (c) S. aureus (ATCC 29213). 
concentration of $\mathrm{COOH}$ groups (15\% AgCLPs) as well as $\mathrm{NaCLPs}$ infused with aqueous $\mathrm{AgNO}_{3}$ (see the Supporting Information) showed a much reduced efficacy, indicating that only the silver present as carboxylates is antibacterially active and that silver infusion of NaCLPs is not as effective as AgCLP formation from AgLignin. For the Gram-negative P. aeruginosa (Figure $3 \mathrm{~b}$ ), the $\mathrm{Ag}^{+}$concentration in AgCLPs required for high antibacterial efficiency was $20 \mathrm{mg} \mathrm{L}^{-1}$, leading to $94 \%$ inhibition of growth. After exposure of $2.6 \%$ AgCLPs via AgLignin against the Gram-positive S. aureus (Figure 3c), the inhibition of growth reached $96 \%$ at a $\mathrm{Ag}^{+}$equivalent of $20 \mathrm{mg}$ $\mathrm{L}^{-1}$, which was found to be better than the free $\mathrm{AgNO}_{3}$ solution at the same $\mathrm{Ag}^{+}$content $(\sim 59 \%$ of inhibition of growth). This can be ascribed to the antibacterial effect of pure CLPs against $S$. aureus, which showed $\sim 80 \%$ inhibition of growth after $24 \mathrm{~h}$. It is known that lignin possesses antibacterial properties in itself, especially against Gram-positive bacteria, ${ }^{24}$ and in previous studies, this antibacterial effect has been observed with CLPs covalently bound to cellulose ${ }^{25}$ although no significant antibacterial effect against Gram-positive Staphylococcus epidermidis had been seen with previous studies with acid-precipitated lignin nanoparticles without silver infusion. ${ }^{4}$ Thus, the synergetic effect of $\mathrm{Ag}^{+}$and CLPs appeared to be important to reduce the growth of $S$. aureus.

Additionally, the antibacterial activity of AgCLPs coated with PDAC (AgCLPs-PDAC) was also evaluated to compare with the antibacterial activity of non-coated AgCLPs against $E$. coli, P. aeruginosa, and S. aureus (Figure S6). The samples with similar $\mathrm{Ag}^{+}$content were exposed to the different bacterial strains, and the concentration of PDAC-coated AgCLPs and the respective inhibition of growth are detailed in Table S2. Based on the initial findings of Richter et al., we expected AgCLPs to require a cationic polyelectrolyte coating with PDAC to enable a stronger adhesion of the particles to the bacterial cell membranes and produce a higher antibacterial activity. ${ }^{4}$ Surprisingly, the PDAC coating seemed to have little, if any, positive influence on the antibacterial efficacy of the particles.

In contrast to the loosely bound silver in the EbNPs, the silver carboxylate AgCLPs do not release silver without the presence of countercations that can be exchanged with silver in the AgCLPs. Thus, the AgCLPs can be stored as water dispersions for long periods of time without the deterioration of their antibacterial activity.

To some extent, the AgCLPs should be considered an intermediate between the EbNPs and carboxylate silver complexes, ${ }^{26}$ or more specifically silver metal-organic frameworks (AgMOFs), ${ }^{27}$ which similar to AgCLPs are large clusters of silver carboxylates. In the case of carboxylate silver complexes, the molecules are dissolved in water, and in the case of AgMOFs, silver is released as the constituent carboxylates and not through cation exchange.

We have shown that AgCLPs show high and sustained antibacterial activity against Gram-positive and Gram-negative strains, even without a cationic polyelectrolyte coating. Thus, AgCLPs are superior in applications requiring sustained antibacterial activity where the fast release of silver, as observed in the EbNPs, is not beneficial. However, we acknowledge that time-limited antimicrobial action can be beneficial in applications with a possibility of bioaccumulation of lignin nanoparticles. In this sense, the two preparation methods of silver-infused lignin nanoparticles can be considered complementary.

\section{CONCLUSIONS}

We have shown that silver can be bound to lignin with deprotonation and ion exchange and that the silver-exchanged lignin is self-assembled into AgCLPs in a suitable process for full solvent recovery and reuse. Due to the ionic bonding of the silver carboxylate groups, they afford high stability of the particles in deionized water. However, the high antibacterial activity of the AgCLPs upon contact with bacteria in physiological conditions indicates that silver is released in the presence of countercations. The formation of silver phenoxide moieties in lignin is also possible in anhydrous media. However, upon contact with water, silver phenoxide moieties hydrolyze into phenol and silver hydroxide, which further condenses into silver oxide NPs. Silver present as carboxylates is both stable and provides sustained antibacterial activity. Additionally, the silver infusion of NaCLPs is possible but does not seem to be as effective as the preparation of AgCLPs from organic solutions of silver carboxylate lignin. Rather surprisingly, no cationic polyelectrolyte coating was required for good antibacterial activity of the AgCLPs. The method of AgCLP production is applicable to the closed-cycle polarity exchange process of CLP formation, which we have introduced. The only added criterion to the process is that whereas the general CLP process is tolerant of water in the recovered solvents, the production of AgCLPs requires anhydrous solvents. As the AgCLPs are stable in distilled water but release silver in physiological conditions, their applicability is greatly enhanced over silver-infused lignin nanoparticles where the silver ions are released immediately after preparation.

\section{ASSOCIATED CONTENT}

\section{Supporting Information}

The Supporting Information is available free of charge on the ACS Publications website at DOI: 10.1021/acssuschemeng.9b02498.

EDS-TEM figures, DLS graphs, description of the AgISE and additional Ag-ISE experiments, antimicrobial results with alternative AgCLP formulations, FT-IR spectra of AgLignin versus neat lignin, XPS measurements of AgLignin, and acid-base-acid titration of CLPs (PDF)

\section{AUTHOR INFORMATION}

\section{Corresponding Author}

*E-mail: mauri.kostiainen@aalto.fi.

ORCID

Kalle Lintinen: 0000-0003-1320-5577

Guillaume N. S. Rivière: 0000-0002-1282-0300

Päivi Tammela: 0000-0003-4697-8066

Monika Österberg: 0000-0002-3558-9172

Hélder A. Santos: 0000-0001-7850-6309

Mauri A. Kostiainen: 0000-0002-8282-2379

\section{Author Contributions}

The manuscript was written through contributions of all authors.

\section{Notes}

The authors declare the following competing financial interest(s): K.L and M.K. declare potential financial interests in the future development and commercialization of the 
antimicrobial AgCLPs. Aalto University has filed a Finnish provisional patent application (FI20195679).

\section{ACKNOWLEDGMENTS}

The financial support through the Academy of Finland (grants 267497,286845 , and 308578 ) is gratefully acknowledged. This work was carried out under the Academy of Finland Centers of Excellence Programme (2014-2019) and made use of the Aalto University Nanomicroscopy Center (Aalto-NMC).

\section{REFERENCES}

(1) Figueiredo, P.; Lintinen, K.; Hirvonen, J. T.; Kostiainen, M. A.; Santos, H. A. Properties and Chemical Modifications of Lignin: Towards Lignin-Based Nanomaterials for Biomedical Applications. Prog. Mater. Sci. 2018, 93, 233-269.

(2) Xu, C.; Arancon, R. A. D.; Labidi, J.; Luque, R. Lignin Depolymerisation Strategies: Towards Valuable Chemicals and Fuels. Chem. Soc. Rev. 2014, 43, 7485-7500.

(3) Sen, S.; Patil, S.; Argyropoulos, D. S. Thermal Properties of Lignin in Copolymers, Blends, and Composites: A Review. Green Chem. 2015, 17, 4862-4887.

(4) Richter, A. P.; Brown, J. S.; Bharti, B.; Wang, A.; Gangwal, S.; Houck, K.; Cohen Hubal, E. A.; Paunov, V. N.; Stoyanov, S. D.; Velev, O. D. An Environmentally Benign Antimicrobial Nanoparticle Based on a Silver-Infused Lignin Core. Nat. Nanotechnol. 2015, 10, 817-823.

(5) Figueiredo, P.; Lintinen, K.; Kiriazis, A.; Hynninen, V.; Liu, Z.; Bauleth-Ramos, T.; Rahikkala, A.; Correia, A.; Kohout, T.; Sarmento, B.; Yli-Kauhaluoma, J.; Hirvonen, J.; Ikkala, O.; Kostiainen, M. A.; Santos, H. A. In Vitro Evaluation of Biodegradable Lignin-Based Nanoparticles for Drug Delivery and Enhanced Antiproliferation Effect in Cancer Cells. Biomaterials 2017, 121, 97-108.

(6) Figueiredo, P.; Ferro, C.; Kemell, M.; Liu, Z.; Kiriazis, A.; Lintinen, K.; Florindo, H. F.; Yli-Kauhaluoma, J.; Hirvonen, J.; Kostiainen, M. A.; Santos, H. A. Functionalization of Carboxylated Lignin Nanoparticles for Targeted and PH-Responsive Delivery of Anticancer Drugs. Nanomedicine 2017, 12, 2581-2596.

(7) Beisl, S.; Miltner, A.; Friedl, A. Lignin from Micro- to Nanosize: Production Methods. Int. J. Mol. Sci. 2017, 18, 1244.

(8) Zhao, W.; Simmons, B.; Singh, S.; Ragauskas, A.; Cheng, G. From Lignin Association to Nano-/Micro-Particle Preparation: Extracting Higher Value of Lignin. Green Chem. 2016, 18, 56935700.

(9) Colman, B. P.; Arnaout, C. L.; Anciaux, S.; Gunsch, C. K.; Hochella, M. F.; Kim, B.; Lowry, G. V.; McGill, B. M.; Reinsch, B. C.; Richardson, C. J.; Unrine, J. M.; Wright, J. P.; Yin, L.; Bernhardt, E. S. Low Concentrations of Silver Nanoparticles in Biosolids Cause Adverse Ecosystem Responses under Realistic Field Scenario. PLoS One 2013, 8, No. e57189, 1-10.

(10) Leskinen, T.; Smyth, M.; Xiao, Y.; Lintinen, K.; Mattinen, M.L.; Kostiainen, M. A.; Oinas, P.; Österberg, M. Scaling Up Production of Colloidal Lignin Particles. Nord. Pulp Pap. Res. J. 2017, 32, 586596.

(11) Lintinen, K.; Xiao, Y.; Bangalore Ashok, R.; Leskinen, T.; Sakarinen, E.; Sipponen, M.; Muhammad, F.; Oinas, P.; Österberg, M.; Kostiainen, M. Closed Cycle Production of Concentrated and Dry Redispersible Colloidal Lignin Particles with a Three Solvent Polarity Exchange Method. Green Chem. 2018, 20, 843-850.

(12) Bangalore Ashok, R. P.; Oinas, P.; Lintinen, K.; Sarwar, G.; Kostiainen, M. A.; Österberg, M. Techno-Economic Assessment for the Large-Scale Production of Colloidal Lignin Particles. Green Chem. 2018, 20, 4911-4919.

(13) Lintinen, K.; Latikka, M.; Sipponen, M. H.; Ras, R. H. A.; Österberg, M.; Kostiainen, M. A. Structural Diversity in Metalorganic Nanoparticles Based on Iron Isopropoxide Treated Lignin. RSC Adv. 2016, 6, 31790-31796.

(14) Nypelö, T. E.; Carrillo, C. A.; Rojas, O. J. Lignin Supracolloids Synthesized from (W/O) Microemulsions: Use in the Interfacial
Stabilization of Pickering Systems and Organic Carriers for Silver Metal. Soft Matter 2015, 11, 2046-2054.

(15) Granata, A.; Argyropoulos, D. S. 2-Chloro-4,4,5,5-Tetramethyl1,3,2-Dioxaphospholane, a Reagent for the Accurate Determination of the Uncondensed and Condensed Phenolic Moieties in Lignins. J. Agric. Food Chem. 1995, 43, 1538-1544.

(16) CLSI Methods for Dilution Antimicrobial Susceptibility Tests for Bacteria That Grow Aerobically: Approved Standard, 2009.

(17) SFS-EN ISO 11885 (2009) Water Quality. Determination of Selected Elements by Inductively Coupled Plasma Optical Emission Spectrometry (ICP-OES) (ISO 11885:2009); Finnish Standards Association SFS: Helsinki.

(18) Kihlman, J. Acid Precipitation Lignin Removal Processes Integrated into a Kraft Mill. In NWBC 2015 The 6th Nordic Wood Biorefinery Conference; 2015, pp 402-410.

(19) Lievonen, M.; José Valle-Delgado, J.; Mattinen, M.-L.; Hult, E.L.; Lintinen, K.; Kostiainen, M. A.; Paananen, A.; Szilvay, G. R.; Setälä, H.; Österberg, M. A Simple Process for Lignin Nanoparticle Preparation. Green Chem. 2016, 18, 1416-1422.

(20) Ragnar, M.; Lindgren, C. T.; Nilvebrant, N.-O. pKa-Values of Guaiacyl and Syringyl Phenols Related to Lignin. J. Wood Chem. Technol. 2000, 20, 277-305.

(21) Biedermann, G.; Sillén, L. G.; Lindberg, B.; Dodson, R. M. Studies on the Hydrolysis of Metal Ions. Part 30. A Critical Survey of the Solubility Equilibria of Ag2O. Acta Chem. Scand. 1960, 14, 717725 .

(22) Tomani, P. E. R. The Lignoboost Process. Cellul. Chem. Technol. 2010, 44, 53-58.

(23) Bastús, N. G.; Merkoçi, F.; Piella, J.; Puntes, V. Synthesis of Highly Monodisperse Citrate-Stabilized Silver Nanoparticles of up to $200 \mathrm{Nm}$ : Kinetic Control and Catalytic Properties. Chem. Mater. 2014, 26, 2836-2846.

(24) Dong, X.; Dong, M.; Lu, Y.; Turley, A.; Jin, T.; Wu, C. Antimicrobial and Antioxidant Activities of Lignin from Residue of Corn Stover to Ethanol Production. Ind. Crops Prod. 2011, 34, 16291634.

(25) Paananen, A.; Szilvay, G.; Setälä, H.; Hult, E.-L.; Alakomi, H.-L. Functionalization of Cellulose with Lignin to Produce High-Value Products. WO 2017/089657 Al, 2017.

(26) Azócar, M. I.; Gómez, G.; Levín, P.; Paez, M.; Muñoz, H.; Dinamarca, N. Review: Antibacterial Behavior of Carboxylate Silver(I) Complexes. J. Coord. Chem. 2014, 67, 3840-3853.

(27) Lu, X.; Ye, J.; Zhang, D.; Xie, R.; Bogale, R. F.; Sun, Y.; Zhao, L.; Zhao, Q.; Ning, G. Silver Carboxylate Metal-organic Frameworks with Highly Antibacterial Activity and Biocompatibility. J. Inorg. Biochem. 2014, 138, 114-121. 